\title{
Electric Field-Induced Gas Emission From PVDF Films
}

\author{
E. Bihler, K. Holdik and W. Eisenmenger
}

\author{
Physikalisches Institut \\ University of Stuttgart \\ Germany
}

\begin{abstract}
Charge injection, conduction and trapping are important processes for stabilizing the electric polarization in the piezoelectric polymer PVDF. In order to study the nature of the pertinent charges in PVDF we measured the gas emission from PVDF using a permeable electrode under an applied electric field up to $0.7 \mathrm{MV} / \mathrm{cm}$. The films were covered on one side with evaporated copper, on the other side a copper wire gauze was used as a permeable electrode. The polymer films were mounted in an UHV-system with a built-in quadrupole mass spectrometer for residual gas analysis. Charging the permeable electrode negatively, gas emission was found mainly consisting of hydrogen, hydrogen fluoride, and fluorine. For comparison FEP- and PET-films were examined.
\end{abstract}

\section{INTRODUCTION}

The conduction mechanisms in insulating polymers can be studied by means of the pressure and temperature dependence of the electric current [1]. We observed a significant reduction of the conductivity [2] (10\% conductivity at $107 \mathrm{~Pa}$ ) in PVDF(poly(vinylidene fluoride)) with increasing pressure indicating ionic charge transport with an activation volume of 0.025 to $0.1 \mathrm{~nm}^{3}$.

A more direct identification of ionic charge carriers is possible by studying the emission of gas atoms from polymers under electric field. For nylon 66 films $\mathrm{H}_{2}$ emission corresponding to the electric field has been observed [3]. A related but more sensitive method, which discriminates the influence of the electric polarity, is used in the present paper to obtain information about the ionic charge transport in PVDF. The charge transport is an important process during formation (polarization) of piezoelectric PVDF-films [4] .

\section{EXPERIMENTAL PROCEDURE}

Polymer films of a thickness of 25 to $50 \mu \mathrm{m}$ were mounted in an UHV-system with a built-in quadrupole mass spectrometer for residual gas analysis (Fig. 1).

The vacuum system uses a diffusion pump with a liquid $\mathrm{N}_{2}$ baffle. After baking the system to $380 \mathrm{~K}$ a residual pressure of about $3 \times 1 \overline{0}^{5} \mathrm{~Pa}$ was obtained.

The films were covered on one side with evaporated copper electrodes (thickness $150 \mathrm{~nm}$, area $20 \mathrm{~cm}^{2}$ ). On

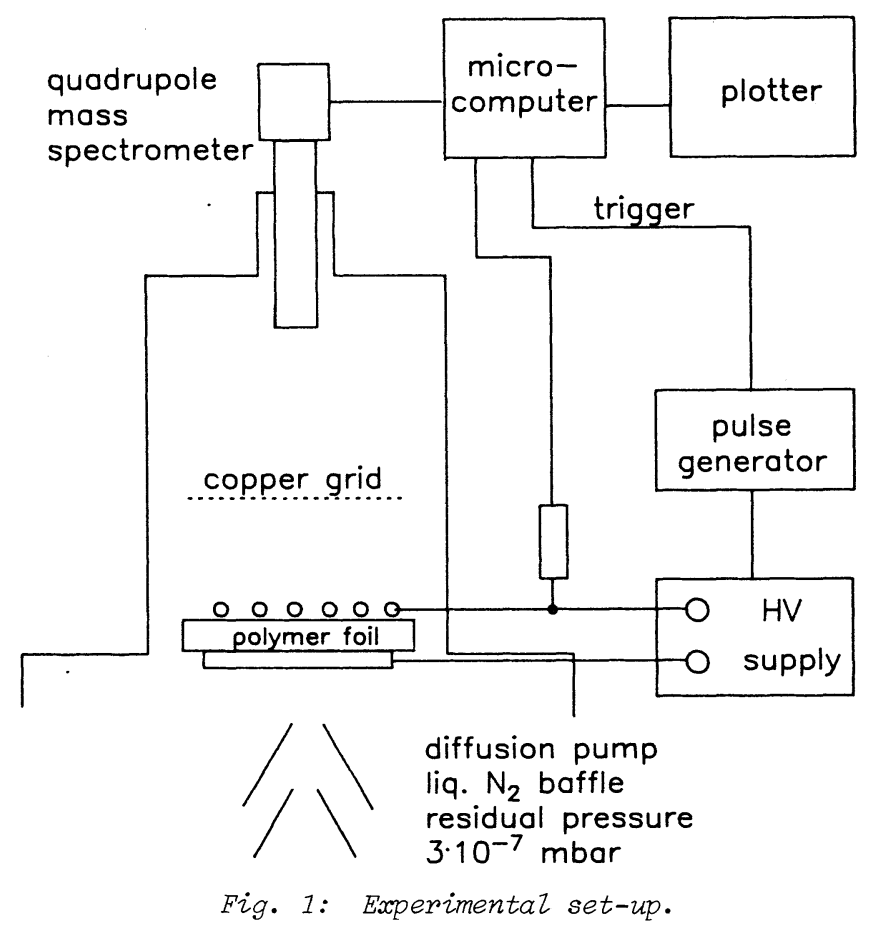

the other side a copper wire gauze (mesh-width approx. $90 \mu \mathrm{m}$, aperture $48 \%$ ) was used as a permeable electrode which faces towards the mass spectrometer. With this 
spectrometer (Balzers QMG 111) a detection of changes in the residual gas composition is possible with a sensitivity of $2 \times 10^{-6} \mathrm{~Pa}$ (according to an ion current of $5 \times 10^{-12} \mathrm{~A}$ ).

An additional electric potential could be applied to a copper grid installed between the polymer foil and the mass spectrometer in order to probe the charge state of the emitted particles.

In order to reduce saturation influences on the gas emission, the electric field ranging from 0 to $1 \mathrm{MV} / \mathrm{cm}$ was applied in almost triangular pulse form of a few $100 \mathrm{~ms}$ duration. Therefore the HV supply was externally controlled by a pulse generator. The voltage pulse and the ion current of a specific mass were digitized by a microcomputer and afterwards plotted. Fig. 2 shows the applied voltage pulse (a) and the gas emmission response (b) of hydrogen at the negatively poled electrode.

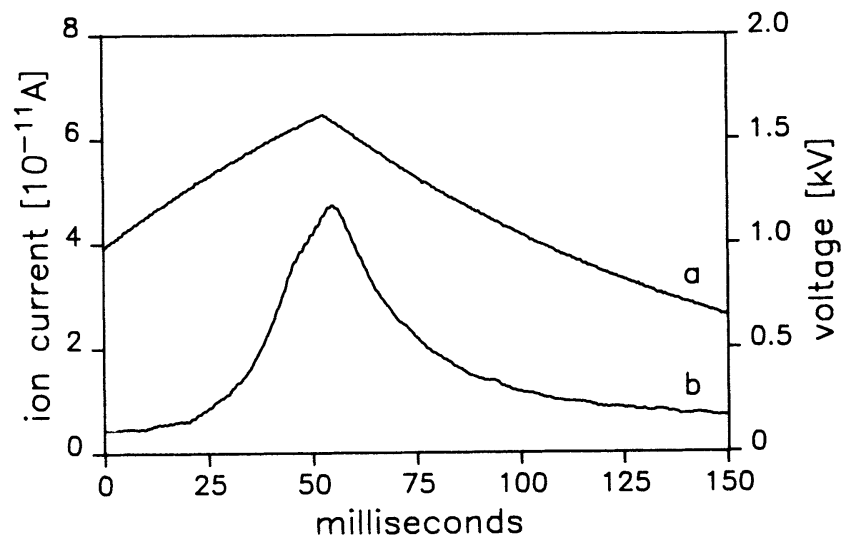

Fig. 2: Voltage pulse of negative polarity at the permeable electrode (a), and gas emission signal for hydrogen of a PVDF scomple (b).

\section{SAMPLE MATERIAL}

We investigated polymer films of PVDF delivered from Dynamit Nobel AG (Trevidur DYFLOR 2000) of $30 \mu \mathrm{m}$ thickness. This unstretched material was found to be totally of Form II by IR-spectroscopy with a crystallinity of about $50 \%$.

The FEP-samples were Teflon®-FEP from DuPont de Nemours with a thickness of $25 \mu \mathrm{m}$ (kindly supported by Dr. Gerhard-Multhaupt). As PET-films we used Hostaphan$R$ (from Kalle $A G$ ). The thickness of these films was $25 \mu \mathrm{m}$.

\section{EXPERIMENTAL RESULTS}

PVDF-films emit gases predominantly at the negatively charged permeable electrode during the polarization with electric fields 0.4 to $1 \mathrm{MV} / \mathrm{cm}$. Fig. 3 shows the hydrogen gas emission as a function of the applied electric field strength. The observed gas emission exhibits threshold field of approx. $0.45 \mathrm{MV} / \mathrm{cm}$. It is to be noted that within our experimental accuracy of approx. $10 \%$ we found the threshold field for the gas emission to coincide with the threshold field for the permanent polarization in PVDF.

Under constant electric field strength, the gas emmission decreases by one order of magnitude within a

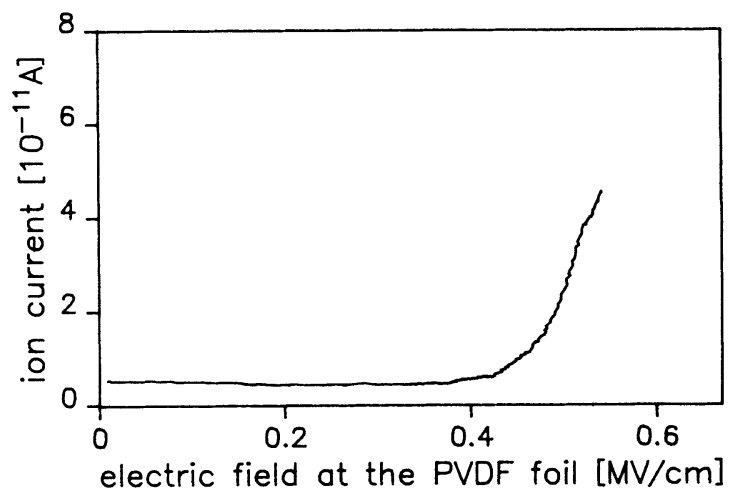

Fig. 3: Hydrogen gas emission versus electric field.

few $10 \mathrm{~s}$. After subsequent $200 \mathrm{~s}$ zero field condition, the gas emission can be observed as before. This "recovery time" can be reduced by increasing the temperature of the sample. The observed recovery time agrees in magnitude with the dielectric relaxation time of 100 $s$ in PVDF. This is another indication for a close relation to the charge transport dynamics in the sample.

Fig. 4a shows the mass distribution of the emitted particles with large concentrations of $\mathrm{H}_{2}, \mathrm{H}$, and $\mathrm{HF}$. The total gas flow can be estimated from the pumping

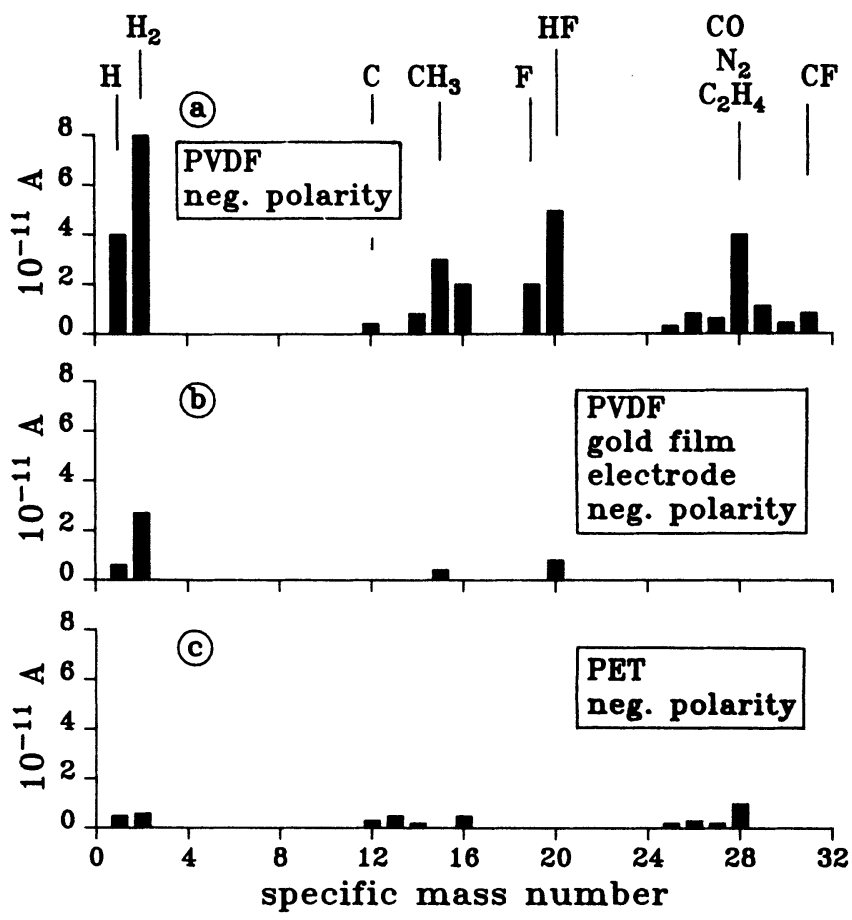

Fig. 4: Mass distributions of field-induced gas emission from PVDF and PET with negative polarity at the permeable electrode.

speed, pumping area and the observed partial pressure to be $10^{14}$ particles/s within one order of magnitude. This compares well with a current of $10^{14}$ elementary charges per second during the polarization procedure of PVDF.

Any electrical potential applied to the grid above the polymer film does not influence the gas emission. This 
is consistent with the emission of neutral gas molecules/atoms. The emission of ionic particles from inside the polymer film would require an electrostatic energy of the order of several eV. This energy is not available.

In order to study the influence of the permeable electrode structure, additional experiments with thin gold films were performed. The evaporated gold films had a thickness of about $20 \mathrm{~nm}$. Such thin films grow inhomogeneously and exhibit small holes and channels.

The use of evaporated electrodes is advantageous because such electrodes providea more intimate contact with the sample surface than a wire mesh where a finite vacuum field in the partly open space between the wires and the sample cannot be avoided.

The experimental result (Fig. 4b) agrees essentially with Fig. 4a and indicates a reduced gas emission, possibly caused by covering more of the polymer surface.

For comparison also the gas emission from PET films (Fig. 4c) and FEP films (not shown) was analyzed. PET shows one order of magnitude reduced gas emission (with the gauze electrode) without HF and F contributions. Typical fragments of the polymer chain are emitted. No gas emission can be observed for FEP.

Experiments with reversed polarity show a drastic change of the gas emission especially for PVDF (Fig. $5 \mathrm{a}$ and $5 \mathrm{~b})$. We observe only $\mathrm{H}_{2}$ and $\mathrm{H}$ emission, reduced in pressure by one order of magnitude.
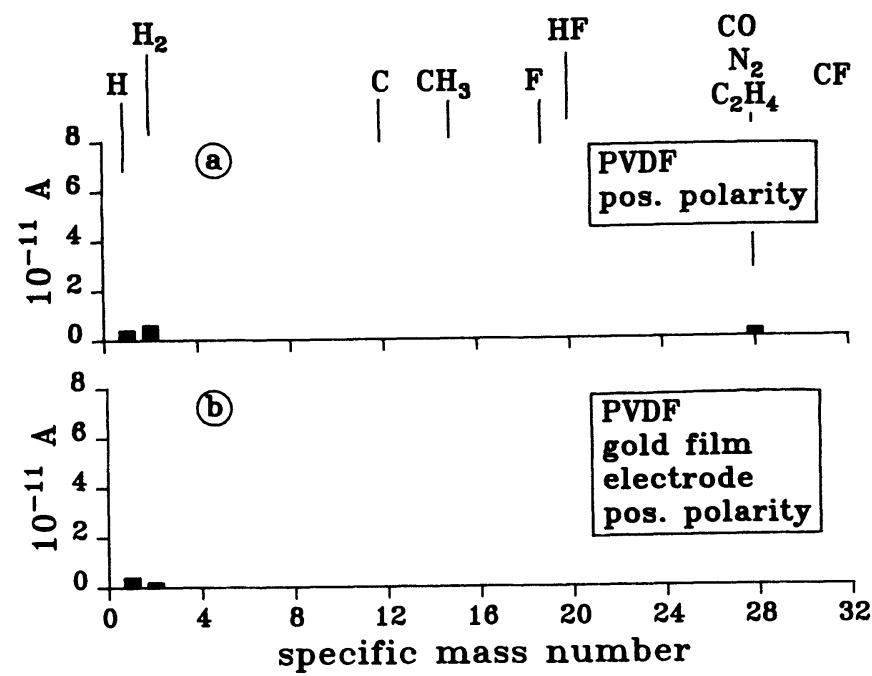

Fig. 5: Mass distributions of field-induced gas emission from PVDF with positive polarity at the permeable electrode.

\section{DISCUSSION AND TENTATIVE MODEL}

Summarizing our results the gas emission can be characterized as follows:

The mass distribution of the emitted particles corresponds to the chemical constituents of the polymer. Typical fragments of the polymer chains are emitted.

The conductivity, the dielectric permittivity, and the magnitude of the gas emission of the studied polymers are correlated:

\begin{tabular}{|c|c|c|c|}
\hline & $\begin{array}{c}\text { conductivity } \\
\mathrm{S} / \mathrm{cm} \\
\end{array}$ & $\begin{array}{c}\text { relative } \\
\text { permittivity }\end{array}$ & $\begin{array}{c}\text { gas flow } \\
\mathrm{s}^{-1}\end{array}$ \\
\hline FEP & $10^{-18}$ & 2.15 & $<10^{12}$ \\
\hline PET & $10^{-16}$ & 3.5 & $10^{13}$ \\
\hline DF & $10^{-14}$ & 9.8 & $10^{14}$ \\
\hline
\end{tabular}

With increasing permittivity or transverse dipolar moment the conductivity and the observed gas emission increases. The fast response of the gas emission to the electrical pulse (within milliseconds) excludes thermal influences. This is consistent with the significant polarity dependence and the estimated maximum possible temperature increase of only $10 \mathrm{mK}$ of the sample, assuming that the total polarization energy is dissipated as heat.

Only atoms and molecules, which are generated in a small surface layer of a few $10 \mathrm{~nm}$ depth, can contribute to the gas emission signal within the observed response time.

Ionization of the polymer by accelerated electrons can be excluded. For the cold emission of electrons from the copper grid electrode electric field above 10 $\mathrm{MV} / \mathrm{cm}$ are necessary, whereas $1 \mathrm{MV} / \mathrm{cm}$ was the maximum field strength applied to the polymer films. Therefore only direct contact of the electrode structure to the polymer surface can lead to charge injection.

Ionic electrode reactions attributed to the polarity of the molecular bonds may explain the observed results:

Electrons (or holes) are injected by tunneling under high field strength creating charged localized states of the polymer chain. Considering the transversal charge displacement in PVDF chains, it should be possible, that the charged states become stabilized by splitting off an ion.

Fig. 6 shows one example of the conceivable reactions at the cathode. It appears plausible that electron

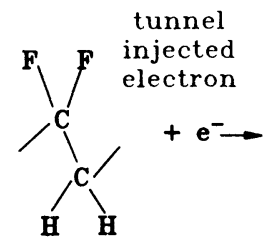

segment of polymer chain

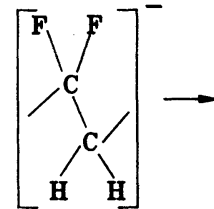
state charged

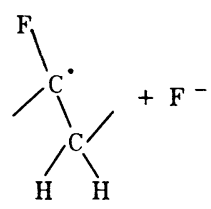

split off reaction
Fig. 6: Model for a possible ionic electrode reaction.

injection causes electronegative atoms to separate from the chain whereas electron extraction leads to separation of electropositive atoms. Since the direct emission of ions from the sample is energetically less favorable, neutralization of the ions by counterions from the other electrode or by countercharges (electrons/holes) is required for the gas emission.

Additional electrode reactions are imaginable, as for example, the production of neutral chain fragments together with the generation of single ions or charges bound to other parts of the polymer chain. 
One part of the generated ions contribute to the bulk charge transport. The other part is emitted after neutralization at the generating electrode.

For polar bonds, as in PVDF, the activation energy for the separation is generally lower than in polymers as PET or FEP with less polar bonds. This explains the relatively strong electric field induced gas emission in PVDF.

\section{SUMMARY}

The observed gas emission of polymer films (PVDF, PET and Teflon-FEP) is consistent with the proposed ionic electrode reaction model. Tunnel injected electrons form charged states, which can stabilize by splitting off ions or uncharged fragments of the polymer chain.

The observed electric field and time dependence of the gas emission from PVDF appears to correlate with charge injection and the formation of permanent polarization.

The ions, generated at the electrodes can contribute to the ionic charge transport observed in PVDF by other experiments and stabilize the polarization by being trapped at the border of polarization zones.

Further studies of the gas emission from PVDF-films under different conditions of temperature and electric field may be useful in order to improve the understanding of charge generation and transport in PVDF.

\section{REFERENCES}

[1] S. Saito, H. Sasabe, T. Nakajiama and K. Yada, "Dielectric Relaxation and Electrical Conduction of Polymers as a Function of Pressure and Temperature", J. Polym. Sci. A2, Vo1. 6, pp. $1297-1315,1968$.

[2] J. Kringler, "Pressure Dependence of Conductivity in PVDF", Diploma Thesis, Phys. Inst. Y1677, 1982, Univ. Stuttgart. (unpublished)

[3] D. A. Seanor, "Electronic and Ionic Conductivity in Nylon 66", J. Poly. Sci. A2, Vol. 2, pp. 463$477,1968$.

[4] W. Eisenmenger and M. Haardt, "Observation of Charge Compensated Polarization Zones in Polyvinylidenefluoride", Solid State Comm., Vol. 41, pp. $917-920,1982$.

बRegistered Trade Mark

Manuscript was received 7 November 1986, in revised form 10 February 1987. 